\title{
Symbolic control of visual attention: Semantic constraints on the spatial distribution of attention
}

\author{
BRADLEY S. GibSON \\ University of Notre Dame, Notre Dame, Indiana \\ Matthias Scheutz \\ Indiana University, Bloomington, Indiana \\ AND \\ Gregory J. DaVIS \\ University of Notre Dame, Notre Dame, Indiana
}

\begin{abstract}
Humans routinely use spatial language to control the spatial distribution of attention. In so doing, spatial information may be communicated from one individual to another across opposing frames of reference, which in turn can lead to inconsistent mappings between symbols and directions (or locations). These inconsistencies may have important implications for the symbolic control of attention because they can be translated into differences in cue validity, a manipulation that is known to influence the focus of attention. This differential validity hypothesis was tested in Experiment 1 by comparing spatial word cues that were predicted to have high learned spatial validity ("above/below") and low learned spatial validity ("left/right"). Consistent with this prediction, when two measures of selective attention were used, the results indicated that attention was less focused in response to "left/right" cues than in response to "above/below" cues, even when the actual validity of each of the cues was equal. In addition, Experiment 2 predicted that spatial words such as "left/right" would have lower spatial validity than would other directional symbols that specify direction along the horizontal axis, such as “ $\rightarrow$ " cues. The results were also consistent with this hypothesis. Altogether, the present findings demonstrate important semantic-based constraints on the spatial distribution of attention.
\end{abstract}

Speakers often use spatial language to orient the attention of listeners to relevant locations and objects in the environment (Clark, 1973; Coventry \& Garrod, 2004); and, empirical studies confirm that spatial terms such as "above," "below," "left," and "right" can reliably elicit both voluntary and involuntary shifts of attention to cued locations (Gibson \& Kingstone, 2006; Ho \& Spence, 2006; Hommel, Pratt, Colzato, \& Godijn, 2001; Logan, 1995; Mayer \& Kosson, 2004; Vecera \& Rizzo, 2004). Findings suggesting that spatial terms can elicit both voluntary and involuntary shifts of attention to cued locations are especially important because they reveal that previously learned linguistic meanings can control the spatial distribution of attention more or less automatically (cf. Jonides, 1981). Given the powerful influence of spatial semantics on attentional control, further research is needed to provide a more complete understanding of the nature of these semantic constraints.

In the present study, we attempt to extend our understanding of linguistic control by considering how differences in the statistical structure of spatial semantics may influence the spatial distribution of attention. Of particular interest is the ambiguity that often arises when spatial terms are used to direct attention. In particular, ambiguity often arises because spatial terms such as "above," "below," "left," and "right" can be defined with respect to a variety of different frames of reference, which may not all be compatible (Carlson, 2003; Coventry \& Garrod, 2004; Kemmerer, 2006; Levinson, 2003; Logan, 1995); in addition, the spatial perspective of the speaker is often different from the spatial perspective of the listener (Schober, 1993, 1995).

Moreover, the meanings of some spatial terms, such as "left" and "right," tend to be more ambiguous than the meanings of other spatial terms,such as "above" and "below," especially when they are defined with respect to the egocentric perspective of the speaker, and the perspective of the speaker is different from the perspective of the listener. In the extreme, spatial terms such as "left" may actually mean right (and vice versa) when the word is uttered from the speaker's egocentric perspective and interpreted from the listener's egocentric perspective and the two are offset by $180^{\circ}$. In contrast, no such ambiguity arises for "above" and "below" in this context so long as the two individuals remain in their upright, canonical orientations.

Previous studies of linguistic control have not explicitly considered how the relative ambiguity of different spatial

B.S. Gibson, bgibson@nd.edu 
terms that are encountered in typical discourse contexts may come to influence the spatial distribution of attention. Such differences in ambiguity may have important implications for attention, because they may translate into learned differences in cue validity - the extent to which a spatial cue provides accurate information about the location of a target object. Indeed, it is well known that differences in cue validity can influence the focus of attention at a location (Johnson \& Yantis, 1995; for reviews, see Pashler, 1998; van der Heijden, 1992). Hence, spatial terms may be more or less effective at focusing the spatial distribution of attention, depending on the consistency with which they have been uttered and interpreted across different perspectives over time.

One consequence of this differential validity hypothesis is that attention may be less focused along the horizontal axis in response to "left/right" cues than it is along the vertical axis in response to "above/below" cues, even in experimental contexts in which the spatial information provided by each of these cues is $100 \%$ valid. Such findings would be theoretically important because they would extend our understanding of linguistic control by showing that the allocation of selective attention is sensitive to the statistical structure of semantic constraints.

Previous studies of linguistic control have used two distinct measures of visual selective attention; however, to date, neither approach has provided evidence capable of addressing the main prediction of the differential validity hypothesis - namely, that attention may be less focused along the horizontal axis in response to "left/ right" cues than it is along the vertical axis in response to "above/"below" cues. The possibility that semantic processing may influence the spatial distribution of attention was first investigated by Logan $(1994,1995,1996$; Logan \& Sadler, 1996), who focused on elementary spatial relations such as above, below, left, and right. Logan (1995) studied how corresponding linguistic terms influenced the spatial distribution of attention by using the spatial-cuing paradigm depicted in the top panel of Figure 1. Observers were required to discriminate the color (red or green) of a circle that had been cued by one of four spatial cues on each trial ("above," "below," "left," or "right"). The cues were $100 \%$ valid and appeared simultaneously with the target display ( 0 -msec cue-target stimulus onset asynchrony [SOA]); in fact, the correct target could not be identified without the aid of the cue because the target display always contained two red circles and two green circles. Logan (1995) argued that the use of 100\% valid cues and ambiguous target displays was important for methodological reasons, because it ensured that the spatial information provided by each of the word cues would be processed equally on each trial.

Logan (1995) found that response times (RTs) for discriminating the color of the cued circle were significantly longer when attention was directed by "left" and "right" cues than when it was directed by "above" and "below" cues (see also Gibson \& Kingstone, 2006; Logan, 1996). However, these findings do not provide strong evidence for the differential validity hypothesis, because they do not reveal whether attention was less focused at the left and right cued locations than at the above and below cued locations. Instead, Logan's (1995) findings are consistent with a variety of explanations that involve differences in the speed with which spatial directions are apprehended or accessed across the two axes. For instance, directions specified along the vertical axis may be accessed more quickly than directions specified along the horizontal axis because the vertical axis has primacy over the horizontal axis, as has been shown in a variety of different tasks ranging from mental rotation to visual search (Bryant, Tversky, \& Franklin, 1992; Corballis, 1988; Franklin \& Tversky, 1990; Logan, 1994). However, differences in the speed with which directions are accessed along the vertical and horizontal axes need not have any implications for the spatial distribution of attention across these two axes.

One way to assess the differential validity hypothesis within Logan's (1995) original spatial-cuing paradigm is to measure whether the ability to discriminate the color of the cued circle is influenced by the color of the opposite circle (i.e., the circle that appears at the location that is opposite the cued circle). If so, performance should be slower and less accurate when the color of the opposite circle is incompatible, rather than compatible, with the color of the cued circle. We will refer to the performance difference observed between the incompatible and compatible conditions as the opposite compatibility effect in the remainder of this article. If "left/right" cues function as if they are less valid than "above/below" cues in this context (even though they are, in fact, equally valid), attention may be less focused at the left/right cued location than it is at the above/below cued location. Consequently, a larger opposite compatibility effect should occur in response to "left/ right" cues than in response to "above/below" cues.

Perhaps the most common way to measure the focus of attention is to estimate the so-called costs-plus-benefits of attention (Jonides, 1981; Jonides \& Mack, 1984; Posner, 1980; Posner, Snyder, \& Davidson, 1980). In general, studies that have estimated costs-plus-benefits have used

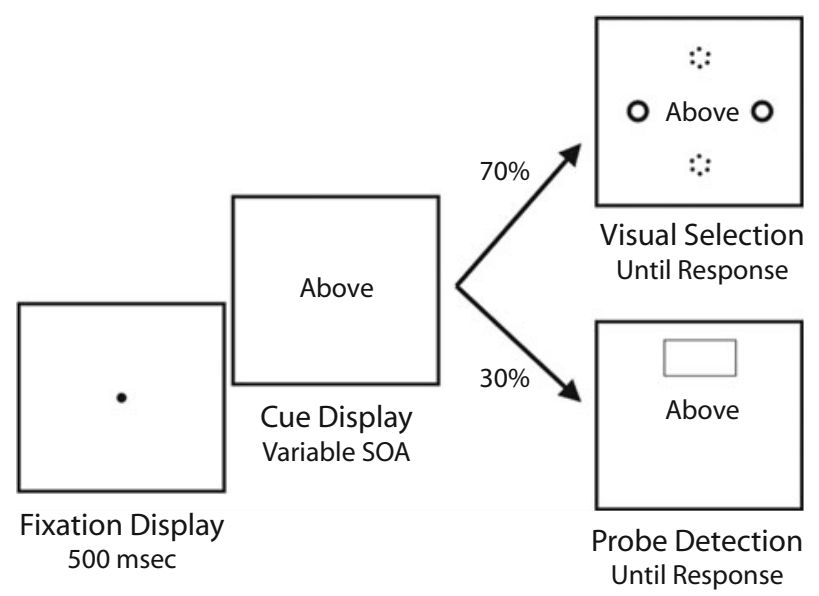

Figure 1. A typical trial sequence used in Experiment 1. The top target display depicts the visual selection task, and the bottom target display depicts the probe detection task. Note that the displays are not drawn to scale. Note also that the dotted lines depict red circles and the solid lines depict green circles. 
target displays in which the identity of the targets is unambiguous and the validity of the cues is less than $100 \%$, resulting in trials in which the target sometimes appears at the cued location (valid trials) and sometimes at uncued locations (invalid trials). In this way, costs-plus-benefits are estimated via a cue validity effect (measured in terms of the difference between the invalid and the valid RTs).

Recall that an estimate of costs-plus-benefits was not possible in Logan's (1995) original paradigm, because he was concerned that such a measure might be underestimated when unambiguous target displays were used, due to the fact that the target can be processed without the aid of the cue under these conditions. Accordingly, the target displays were always ambiguous and the spatial word cues were always 100\% valid in Logan's (1995) experiments (see also Gibson \& Kingstone, 2006).

Consequently, very little is known about the efficacy of spatial word cues in generating cue validity effects and, especially, about whether such effects might be larger for "above/below" cues than for "left/right" cues, reflecting a difference in the focus of attentional resources. Mayer and Kosson (2004) used both auditory- and visuospatial word cues that were $75 \%$ valid in a target detection task and found robust cue validity effects, regardless of cue modality, across cue-target SOAs of 400, 500, and $800 \mathrm{msec}$ (see also Vecera \& Rizzo, 2004). Likewise, Hommel et al. (2001) used uninformative spatial word cues and found robust cue validity effects across a variety of target detection and discrimination tasks, as well as across a variety of cue-target SOAs (see also Ho \& Spence, 2006). These studies provide evidence, based on the presence of significant cue validity effects, that spatial word cues may elicit both voluntary and involuntary shifts of attention to the cued location.

However, none of these previous studies have examined whether costs-plus-benefits are greater for "above/ below" cues than for "left/right" cues. Obtaining a larger cue validity effect for "above/below" cues than for "left/ right" cues would thus provide important converging evidence that greater attentional resources are allocated to the above/below cued locations than to the left/right cued locations, as would be expected if "above/below" cues function as if they are more valid than "left/right" cues.

\section{EXPERIMENT 1}

In the present study, we investigated the extent to which "above/below" and "left/right" cues influenced two measures of selective attention by randomly mixing both ambiguous and unambiguous target displays within the same experiment. The ambiguous displays were identical to those that have been used in previous studies of the cued location effect (Gibson \& Kingstone, 2006; Logan, 1995; see also Figure 1, top panel) and were presented on $70 \%$ of the trials in the present study. On these trials, observers were required to discriminate the color of the cued circle. Because the word cues were essential in performing this visual selection task, we expected that the observers would routinely process the meaning of the cues on each trial and would attempt to orient their attention to the cued location
(Logan, 1995). Thus, we expected to observe a cued location effect in the ambiguous target condition (Gibson \& Kingstone, 2006; Logan, 1995). More important, we also examined whether the compatibility of the opposite circle influenced responses to the cued circle differently across the horizontal and vertical axes, as would be expected if the previously learned differences in cue validity continued to have consequences for the efficiency of selective attention. In addition, we investigated the time course of these effects by including cue-target SOAs of 0,500 , 1,000 , and $1,500 \mathrm{msec}$.

Unambiguous target displays appeared on the remaining $30 \%$ of the trials and were created by presenting a single gray square (instead of the four colored circles) at one of the four cardinal locations in the visual periphery or at fixation. On these trials, thte observers were required to simply press either response key as soon as they detected the presence of the gray square. Unlike in the visual selection task, the probe target appeared at the cued location only by chance (i.e., on one fifth of the trials). This arrangement allowed us to examine probe detection RTs in four cue validity conditions: a valid condition in which the probe happened to appear at the cued location; an invalid-opposite condition in which the probe happened to appear at the location that was opposite to the cued circle; an invalidorthogonal condition in which the probe happened to appear at one of the two locations that were orthogonal to the cued circle; and an invalid-fixation condition in which the probe happened to appear (around the cue) at fixation. Note that although the target appeared at the cued location only by chance in the probe detection task, we nevertheless assumed that the observers would be motivated to shift their attention to the cued location, given that the cues were necessary when the ambiguous target displays were shown unpredictably on $70 \%$ of the trials.

Comparison of probe detection RTs across these four cue validity conditions allowed us to examine whether the magnitude of the cue validity effect would be larger in the above/below cued location condition than in the left/right cued location condition, as would be expected if learned differences in the validity of the cues influenced the focus of attention along the cued axis. If so, further examination may also reveal whether valid RTs would be significantly shorter in the above/below condition than in the left/right condition, and/or whether invalid RTs would also be significantly longer in the above/below condition than in the left/right condition. Either or both of these findings would be consistent with the notion that attention is more focused at the cued location when direction is specified along the vertical axis than when it is specified along the horizontal axis, due to the differential validity of the corresponding cues.

A question of particular interest was whether probe detection RTs would differ across the three invalid cue conditions. According to the differential validity hypothesis, attention may be less focused along the horizontal axis than it is along the vertical axis. Thus, the likelihood that attentional resources will be allocated to an uncued location that appears along the cued axis should be relatively higher in the leftright cued location condition than in the 
above/below cued location condition. Because the invalidopposite and invalid-fixation conditions both fell along the cued axis, it is possible that these invalid RTs may also vary as a function of cued location, with RTs in the above/ below cued location condition being longer than RTs in the left/right cued location condition, again reflecting greater focus of attention at the cued location. Such a finding would be especially powerful in the invalid-fixation condition, because RTs would differ despite the fact that the same physical location was probed across the two cued location conditions. In contrast, because the invalidorthogonal condition falls off the cued axis, no difference was expected between the two cued location conditions.

\section{Method}

Participants. Thirty-six undergraduates from the University of Notre Dame participated in this experiment in partial fulfillment of a course requirement. All of the observers reported normal color vision and normal or corrected-to-normal acuity.

Stimuli and Apparatus. The experimental methodology was based on the experiments reported by Logan (1995; see also Gibson \& Kingstone, 2006). Three displays were presented on each trial: a fixation display, a cue display, and a target display. The initial fixation display included a small fixation dot $\left(0.38^{\circ}\right.$ in diameter). The cue displays contained one of the four word cues, which replaced the fixation dot when they appeared. The word cues were all $0.68^{\circ}$ tall and ranged in length from $1.18^{\circ}$ to $1.94^{\circ}$. The ambiguous target displays contained four colored $O$ s that measured $1.26^{\circ}$ of visual angle in diameter and were presented at the four cardinal locations, approximately $4.37^{\circ}$ from the central fixation point. Two of the $O$ s were colored red and two were colored green on each trial. The unambiguous target displays contained a single gray square that appeared at the cued location, one of the three remaining uncued peripheral locations, or fixation. The probe stimulus subtended $2.18^{\circ}$ of visual angle on all sides and was large enough to appear around the cue when it appeared at fixation. When the probe stimulus appeared in the periphery, it appeared centered at each of the four locations used in the visual selection task. All the stimuli appeared on a standard CRT monitor's black background. The observers viewed the display at a constant viewing distance of $60 \mathrm{~cm}$, which was enforced by a chinrest. Responses were recorded on a custom-made buttonbox (Lafayette Instruments) and were recorded to the nearest millisecond.

Procedure and Design. A typical trial sequence in the ambiguous target condition is shown in the upper panel of Figure 1. Each trial began with a fixation display for $500 \mathrm{msec}$, followed by the cue display. The cues were presented equally often in each of four cuetarget SOA conditions: $0,500,1,000$, and $1,500 \mathrm{msec}$; each of the four SOA conditions was presented randomly during the experimental trials. Cues stayed on throughout the duration of the trial in order to eliminate any load that might be placed on working memory, as well as to dissuade the observers from using different representational codes to maintain the spatial information conveyed by the cue (Lavie, Hirst, de Fockert, \& Viding, 2004). The observers were instructed to keep their eyes fixated on the fixation dot and the cue throughout each trial. An initial pilot study in which eye movements were monitored with the aid of a video camera (Prinzmetal, McCool, \& Park, 2005) indicated that the observers could easily comply with this fixation instruction across the four SOA conditions used in the present study. The results indicated that observers committed eye movements on only a very small percentage of trials $(M=0.02 \%)$ and that this did not significantly influence performance; therefore, eye movements were not monitored in the main study.

The ambiguous target display appeared following the cue-target SOA and, together with the cue, remained on the screen until a response was made (or until 4,000 msec had elapsed). There were a total of six different target displays, which included all possible arrangements of the two red and two green circles; each cue was paired with each of the six target displays equally often. The cues were $100 \%$ valid and always indicated which one of the four $O$ s was the target; the observers' task was to determine as quickly and as accurately as possible whether the target $O$ was red or green. The cue referred to each one of the four target locations equally often, and on any given trial, each location was equally likely to contain a red or green $O$. In this way, the observers could not determine (without guessing) how to respond without the aid of the cue. These contingencies provided reasonable assurance that the observers would process each of the cues equally. Under these conditions, the color of the opposite circle was incompatible with the color of the cued circle on $67 \%$ of the trials, whereas the color of the opposite circle was compatible with the color of the cued circle on the remaining 33\% of the trials. The observers always used their left hand to respond red and their right hand to respond green; however, for half of the observers, the response pad was arrayed horizontally (with the red key to the left of the green key), and for the other half, the response pad was arrayed vertically (with the red key above the green key).

The trial sequence in the unambiguous target condition was identical to that in the ambiguous target condition, except that the probe display appeared instead of the four colored circles (see Figure 1, bottom panel). On these trials, the observers were instructed to press either response key on the response pad as quickly and accurately as possible when the probe target was presented.

The ambiguous target displays appeared on approximately $70 \%$ of the trials $(n=576)$, and the unambiguous target displays appeared on approximately $30 \%$ of the trials $(n=240)$. The experimental trials were preceded by 20 practice trials ( 16 for the visual selection task performed on ambiguous trials and 4 for the probe detection task performed on unambiguous trials). A different random order was presented to each observer.

\section{Results}

In the present experiment, we investigated whether previously learned differences in the validity of spatial word cues could influence the magnitude of the opposite compatibility and cue validity effects. These effects were measured within the ambiguous and unambiguous target conditions, respectively.

Ambiguous target displays. Mean correct RTs and percentages of errors in the visual selection task are depicted in Figure 2 as a function of cued location, opposite compatibility, and SOA. A $2 \times 2 \times 4$ repeated measures ANOVA was performed on mean correct RTs and percentages of errors separately, with cued location (above/below vs. left/right), opposite compatibility (compatible vs. incompatible), and SOA (0 vs. 500 vs. 1,000 vs. 1,500 msec) as the three within-subjects factors.

An analysis of mean correct RTs provided support for the differential validity hypothesis. As was expected, there was a greater opposite compatibility effect observed in the left/right cued location condition than in the above/below cued location condition; in addition, the magnitude of this opposite compatibility effect decreased as SOA increased. This conclusion was supported by a significant three-way interaction between cued location, opposite compatibility, and SOA $[F(3,105)=2.75, p<$ $\left..05, \eta_{\mathrm{p}}^{2}=.07\right]$.

The significant three-way interaction between cued location, opposite compatibility, and SOA was investigated further by examining the effects of opposite compatibility and SOA within each cued location condition separately. Within the above/below cued location condition, there was a significant main effect of SOA $[F(3,105)=336.08, p<$ 


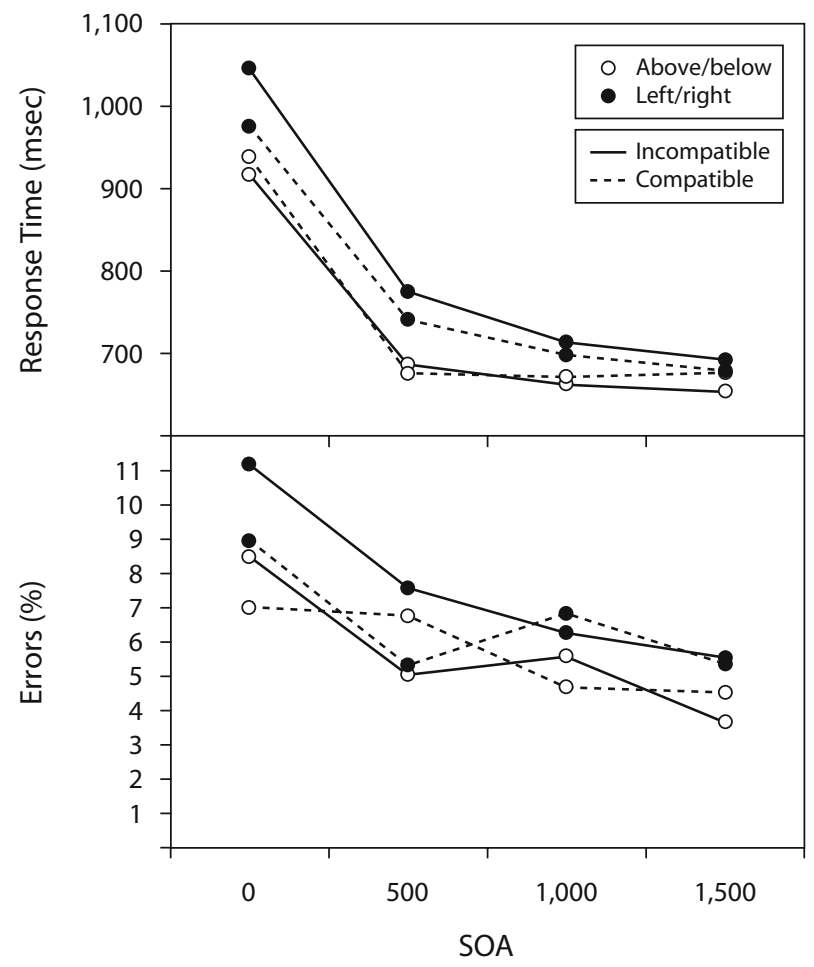

Figure 2. Mean correct response times (top panel) and error rates in percentages (bottom panel) for the visual selection task depicted as function of cued location, opposite compatibility, and stimulus onset asynchrony (SOA) in Experiment 1.

$\left..0001, \eta_{\mathrm{p}}^{2}=.91\right]$, but neither the main effect of opposite compatibility nor the opposite compatibility $\times$ SOA interaction attained significance (both $p$ s $>.05$ ). These results suggest that the color of the opposite circle had no effect on responses to the cued circle when "above/below" cues were shown.

In contrast, the color of the opposite circle had a strong effect on responses to the cued circle when "left/right" cues were shown. There were significant main effects of opposite compatibility $\left[F(1,35)=8.53, p<.01, \eta_{\mathrm{p}}^{2}=\right.$ $.20]$ and $\operatorname{SOA}\left[F(3,105)=184.37, p<.0001, \eta_{\mathrm{p}}^{2}=.84\right]$. In addition, there was a significant opposite compatibility $\times \mathrm{SOA}$ interaction $\left[F(3,105)=3.05, p<.05, \eta_{\mathrm{p}}^{2}=\right.$ $.08]$, indicating that the effect of opposite compatibility decreased as SOA increased. Individual analyses revealed that incompatible RTs were significantly longer than compatible RTs in the 0 - and 500-msec SOA conditions $\left[F(1,35)=15.83, p<.001, \eta_{\mathrm{p}}^{2}=.31\right.$, and $F(1,35)=$ $3.72, p=.06, \eta_{\mathrm{p}}^{2}=.10$, respectively] but not in the 1,000 and 1,500-msec SOA conditions (both $F \mathrm{~s}<1$ ).

An identical three-way repeated measures ANOVA was also performed on error rates (in percentages). Although the pattern of error rates generally mirrored the pattern of mean correct RTs, the statistical analysis revealed significant main effects only of cued location $[F(1,35)=$ $\left.14.04, p<.002, \eta_{\mathrm{p}}^{2}=.29\right]$ and $\operatorname{SOA}[F(3,105)=15.05$, $\left.p<.001, \eta_{\mathrm{p}}^{2}=.30\right]$. Thus, the present pattern of findings obtained in the visual selection task does not appear to reflect a speed-accuracy trade-off.
Unambiguous target displays. Mean correct probe detection RTs are shown in Figure 3 as a function of SOA and cue validity in each of the two cued location conditions separately. Only errors of omission were possible on probe detection trials, and these did not occur in the present experiment. Mean correct RTs were analyzed using a $4 \times 4 \times 2$ repeated measures ANOVA with SOA ( 0 vs. 500 vs. 1,000 vs. $1,500 \mathrm{msec}$ ), cue validity (valid vs. invalid-opposite vs. invalid-orthogonal vs. invalidfixation), and cued location (above/below vs. left/right) as the three within-subjects factors.

An analysis of probe detection RTs also provided support for the differential validity hypothesis. As can be seen in Figure 4, the magnitude of costs-plus-benefits was generally larger in the above/below cued location condition than in the left/right cued location condition. This interpretation was supported by a significant two-way interaction between cue validity and cued location $[F(3,105)=$ $\left.5.29, p<.005, \eta_{\mathrm{p}}^{2}=.13\right]$. However, unlike the results obtained in the visual selection task, the difference in the magnitude of the costs-plus-benefits that was observed across the two cued location conditions remained relatively stable across SOA, as indicated by the nonsignificant three-way interaction between SOA, cue validity, and cued location $(F<1)$.

In order to further evaluate the cue validity $\times$ cued location interaction, costs-plus-benefits were estimated in three separate ways for each of the two cued location conditions: orthogonal RT - valid RT, opposite RT -

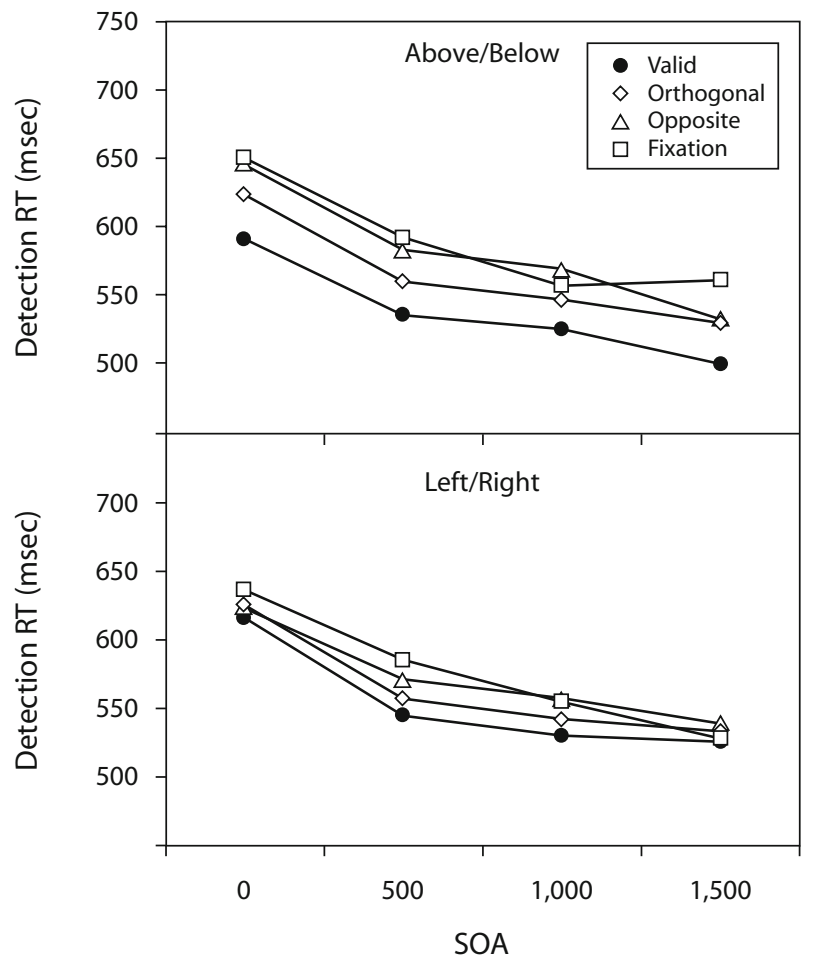

Figure 3. Probe detection response times (RTs) depicted as a function of cue validity and stimulus onset asynchrony (SOA) in each of the "above/below" (top panel) and "left/right" (bottom panel) cued location conditions in Experiment 1. 


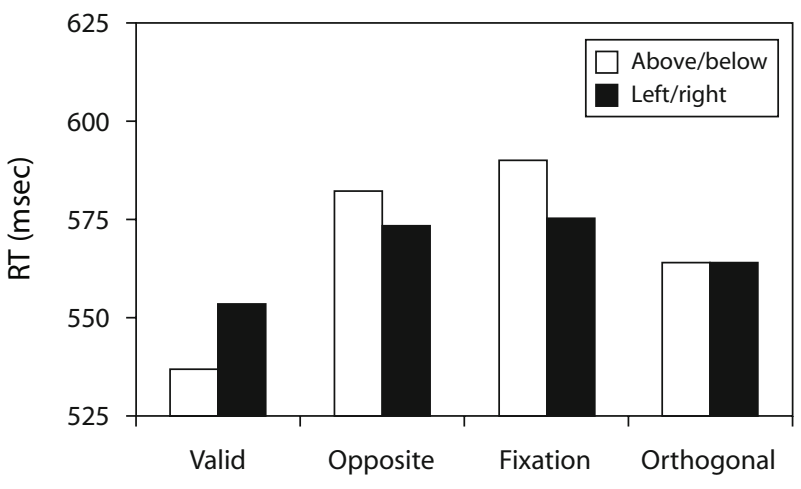

Figure 4. Probe detection response times (RTs) depicted as a function of cue validity and cued location in Experiment 1.

valid RT, and, fixation RT - valid RT. In the orthogonal RT - valid RT condition, the magnitude of the cue validity effect was found to be $27 \mathrm{msec}$ in the above/below cued location condition and $11 \mathrm{msec}$ in the right/left cued location condition. In the opposite RT - valid RT condition, the magnitude of the cue validity effect was found to be $45 \mathrm{msec}$ in the above/below cued location condition and $20 \mathrm{msec}$ in the right/left cued location condition. And in the fixation RT - valid RT condition, the magnitude of the cue validity effect was found to be $53 \mathrm{msec}$ in the above/below cued location condition and $22 \mathrm{msec}$ in the right/left cued location condition. In each case, costs-plusbenefits were found to be significantly larger in the above/ below cued location condition than in the left right cued location condition, as indicated by significant cue validity $X$ cued location interactions within each of these three costs-plus-benefits conditions (all $p$ s $<.025$ ).

As was expected, these differences in costs-plusbenefits occurred because valid RTs were significantly longer in the left/right cued location condition than in the above/below cued location condition. More specifically, valid RTs were, overall, $16 \mathrm{msec}$ longer in the left/right cued location condition than in the above/below cued location condition $\left[F(1,35)=6.17, p<.02, \eta_{\mathrm{p}}^{2}=.15\right.$, for the main effect of cued location], and this effect of cued location did not vary significantly across SOA $(F<1$ for the SOA $\times$ cued location interaction). Consistent with the differential validity hypothesis, this finding suggests that "left/right" cues elicited weaker focus of attention at the cued location than did "above/below" cues.

Further evidence that "left/right" cues elicited a weaker focus of attention at the cued location than did "above/ below" cues was sought by investigating whether RTs in the invalid-opposite and invalid-fixation conditions differed as a function of cued location. Recall that the probe in both of these invalid conditions appeared on the cued axis; thus, RTs were expected to be significantly shorter in the left/right cued location condition than in the above/ below cued location condition, due to differences in the diffusion of attention across the two axes. As was predicted, RTs were, overall, $12 \mathrm{msec}$ shorter in the left/right cued location condition than in the above/below cued lo- cation condition when averaged across these two invalid conditions, although this main effect of cued location attained only marginal significance in the present study $\left[F(1,35)=3.03, p=.09, \eta_{\mathrm{p}}^{2}=.08\right]$.

The pattern of findings observed across the valid, invalid-opposite, and invalid-fixation conditions indicated significant (or marginally significant) differences between the two cued location conditions that were consistent with the differential validity hypothesis. In addition, the differential validity hypothesis predicted that performance should not differ as a function of cued location in the invalid-orthogonal condition. Consistent with this prediction, RTs in the invalid-orthogonal condition were found to be identical across the two cued location conditions and, thus, did not differ significantly $(F<1$ for both the main effect of cued location and the SOA $\times$ cued location interaction).

In summary, the results of Experiment 1 showed greater opposite compatibility effects and smaller cue validity effects when attention was cued to left/right locations than when it was cued to above/below locations. According to the differential validity hypothesis, such differences arise because "left" and "right" cues have previously been experienced as being more ambiguous (less valid) when encountered from opposing perspectives than have "above" and "below" cues. As a result, attention is allocated in a less focused fashion along the horizontal axis than along the vertical axis.

\section{EXPERIMENT 2}

Experiment 2 was conducted to investigate two issues. First, although the spatial validity of each of the word cues was actually equal in Experiment 1, invalid trials did occur on occasion in the probe detection task, which may have had a greater effect on performance in the "left/right" cue condition than in the "above/below" cue condition. We therefore considered it prudent to examine the differential validity hypothesis under conditions in which invalid trials never appeared in the experiment. Accordingly, Experiment 2 used only $100 \%$ valid cues and focused exclusively on the visual selection task.

Second, humans use a variety of directional symbols to control the spatial distribution of attention. Thus, it is important to investigate whether the results observed in Experiment 1 are unique to the spatial word cues that were used in that experiment. A first question concerned whether other spatial words that specify direction along the vertical axis, such as "up/down," can control selective attention as efficiently as "above/below." The comparison of "up/down"and "above/below" cues is also interesting because "up/down" cues express a more basic spatial relation than do "above/below" cues. Logan (1995) suggested that "above/below" cues express a spatial relation between a reference object (cue) and a located object (target), and the direction indicated by this relation can be specified when observers project their frame of reference onto the reference object (see also Carlson, 2003; Coventry \& Garrod, 2004; Kremmerer, 2006; Levinson, 2003). In contrast, the directions indicated by "up/down" cues can 
be specified more directly with respect to the observer's frame of reference, as if the observer himself were the reference object. Thus, the spatial referents associated with "up/down" cues may be determined more quickly than the spatial referents associated with "above/below" cues, because up and down can be specified directly with respect to the observer, whereas above and below first require the observer to project his or her frame of reference onto the reference object (the cue). Consequently, attention may be shifted more quickly in response to "up/down" cues than in response to "above/below" cues, if shifts of attention are influenced by the complexity of the underlying spatial relations.

A second question concerned whether it is possible for other directional symbols to specify direction in a more consistent fashion along the horizontal axis. We propose that there are at least two ways for a symbol to specify direction in a more consistent fashion along the horizontal axis. First, symbols may specify direction in a more consistent fashion along the horizontal axis, despite changes in perspective, by trading spatial ambiguity for perceptual ambiguity. Consider the arrow. Directional arrows are iconic symbols that point to different locations by virtue of their orientation. For instance, a leftward-pointing arrow differs from a rightward-pointing arrow by virtue of a $180^{\circ}$ rotation. Because directional arrows are intended to be iconic symbols, they typically convey direction visually. And because the perceived orientation of a visual stimulus can depend on the perspective of the observer, a single arrow can simultaneously be perceived to be pointing in two directions at once by two different observers.

In the extreme, consider a situation in which two individuals who are offset by $180^{\circ}$ are looking at an arrow. In this situation, the arrow can appear to be pointing in the leftward direction from one perspective at the same time that it can appear to be pointing in the opposite, rightward direction from the other perspective. In other words, the arrow remains spatially valid for both individuals because it can simultaneously convey a location on the left to one individual and a location on the right to the other individual. Consequently, " $\leftarrow$ " and " $\rightarrow$ " cues would be experienced as being more valid than "left" and "right" in situations such as this. If the focus of selection is influenced by these hypothetical differences in learned validity, the opposite compatibility effect should be smaller in response to " $\leftarrow / \rightarrow$ " cues than it is in response to "left/right" cues.

Second, symbols may also specify direction in a more consistent fashion along the horizontal axis if these symbols are encountered only from a single perspective. Because well-learned directional symbols are likely to be encountered from a variety of perspectives outside the laboratory, we investigated this possibility in the present study by training relatively novel associations between words and spatial relations. In particular, observers were instructed to use number words, such as "one," "two," "three," and "four," to refer to the above, right, below, and left relations, respectively.

Although "four" and "two" cues would be just as inconsistent as "left" and "right" cues if these terms were encountered from opposing perspectives, the spatial rela- tions conveyed by the cues in the number word condition were intended to be encountered only from the perspective of the observer in the present experiment. Consequently, "four" and "two" cues should be more valid than "left" and "right" cues in the present experiment. If the focus of selection is influenced by these hypothetical differences in learned validity, the opposite compatibility effect should be smaller in response to "four/two" cues than it is in response to "left/right" cues.

Note that Gibson and Kingstone (2006) recently compared spatial word cues with corresponding arrow cues and found that the RT difference between left/right and above/below cued locations occurred only in the spatial word cue condition. Likewise, Logan (1995) compared spatial word cues with digit cues (as opposed to number word cues) and found that this cued location effect occurred only in the spatial word condition. In both studies, the differential effect of cued location was explained in terms of the complexity of the spatial relations expressed by the directional symbols and the primacy of the vertical axis over the horizontal axis. Recall that spatial word cues are thought to express a spatial relation between a reference object (cue) and a located object (target); in contrast, the spatial relations expressed by corresponding arrow cues and digit cues are thought to be specified more directly with respect to the observer's frame of reference. In this view, the cued location effect arises in the word cue condition because observers have to project their frame of reference onto the reference object and, in so doing, can compute the vertical axis more efficiently than the horizontal axis. However, as was mentioned in the introduction, this accessibility account makes no predictions for the opposite compatibility effect, and neither of these two previous studies examined the opposite compatibility effect across different cue types. Thus, it is currently unknown whether selection is more efficient in response to " $\leftarrow / \rightarrow$ " and "four/two" cues than it is in response to "left/ right" cues.

\section{Method}

Participants. A total of 72 undergraduates from the University of Notre Dame participated in this study in partial fulfillment of a course requirement. Eighteen participants were randomly assigned to each of the four cue conditions: "above/below/left/right," "up/down/ left/right," "one/three/four/two," and " $\uparrow / \downarrow / \leftarrow / \rightarrow$." All of the observers reported normal color vision and normal or corrected-to-normal acuity.

Stimuli and Apparatus. The stimuli and apparatus were identical to those used in Experiment 1, with the sole exception being the inclusion of additional spatial word cues (i.e., "up/down”), arrow cues, and number word cues in Experiment 2. As with the spatial words, the number words were all $0.68^{\circ}$ tall and ranged in length from $1.18^{\circ}$ to $1.94^{\circ}$. Overall, the average word frequency for the four number words $(M=.1397, S D=.13$ ) was estimated (on the basis of Kučera \& Francis, 1967) to be higher than the average word frequency for the four spatial words $(M=.0377, S D=.02)$, but this difference did not attain significance $[t(6)=-1.54, S E=0.07$, $p>.15$, two-tailed]. The arrow cues subtended $0.48^{\circ} \times 1.18^{\circ}$ of visual angle and appeared at each of four orientations, relative to the observer: $\uparrow, \downarrow, \leftarrow$, and $\rightarrow$.

Procedure and Design. The trial sequence was identical to that in Experiment 1. Each of the four cue types was presented to a separate group of observers. The observers in the number word con- 
dition were told that each number cue referred to a specific spatial relation. In particular, the word "one" referred to the above relation, the word "two" referred to the right relation, the word "three" referred to the below relation, and the word "four" referred to the left relation. Within each cue type group, the cues were presented equally often in each of four cue-target SOA conditions: 0,500 , 1,000 , and $1,500 \mathrm{msec}$. There were a total of 576 experimental trials that were preceded by 16 practice trials. All experimental trials were randomized for each observer.

\section{Results and Discussion}

Mean correct RTs and percentage of errors are depicted in Figure 5 as a function of SOA and cued location in each of the four cue type conditions. Mean correct RTs and percentage of errors were analyzed separately using a $4 \times 2 \times 2 \times 4$ mixed ANOVA, with cue type ("above/ below/left/right," "up/down/left/right," "one/three/four/ two," and " $\uparrow / \downarrow / \leftarrow / \rightarrow$ ") as the sole between-subjects factor and cued location, opposite compatibility, and SOA as the three within-subjects factors.

Consistent with Experiment 1, the results of Experiment 2 showed that the opposite compatibility effect observed with mean correct RT was larger in the leftright cued location condition than in either the above/below or the up/down cued location conditions. In addition, the results of Experiment 2 showed that this asymmetry in the magnitude of the opposite compatibility effect occurred in the spatial word condition, but not in the arrow and number word conditions. In support of this conclusion, the cue type $\times$ cued location $\times$ opposite compatibility interaction was found to be significant $[F(2,51)=5.10, p<$ $\left..02, \eta_{\mathrm{p}}^{2}=.17\right]$; however, the cue type $\times$ cued location $\times$ opposite compatibility $\times$ SOA interaction did not attain significance $\left[F(6,153)=1.77, p>.11, \eta_{\mathrm{p}}^{2}=.06\right]$.

The significant three-way interaction between cue type, cued location, and opposite compatibility was further investigated by conducting separate ANOVAs to evaluate the effect of opposite compatibility while holding cued location constant in the various cue type conditions. In the first analysis, we compared the opposite compatibility effect across the two spatial word groups that received "left/ right" cues. As was expected, this analysis revealed a significant main effect of opposite compatibility $[F(1,34)=$ $\left.22.22, p<.001, \eta_{\mathrm{p}}^{2}=.40\right]$, and this effect did not interact with group $\left[F(1,34)=2.32, p>.10, \eta_{\mathrm{p}}^{2}=.06\right]$.

In addition, it is interesting to note that the opposite compatibility effect observed in the two spatial word conditions in Experiment 2 was very similar to the opposite compatibility effect observed in Experiment 1, even though there were no invalid probe trials presented in Experiment 2. Thus, it is unlikely that the inclusion of invalid probe trials exacerbated the magnitude of the opposite location effect observed in the left right cued location condition in Experiment 1. This conclusion was confirmed in an additional analysis in which the effects of opposite compatibility observed in left/right cued location condition were compared across the two experiments.

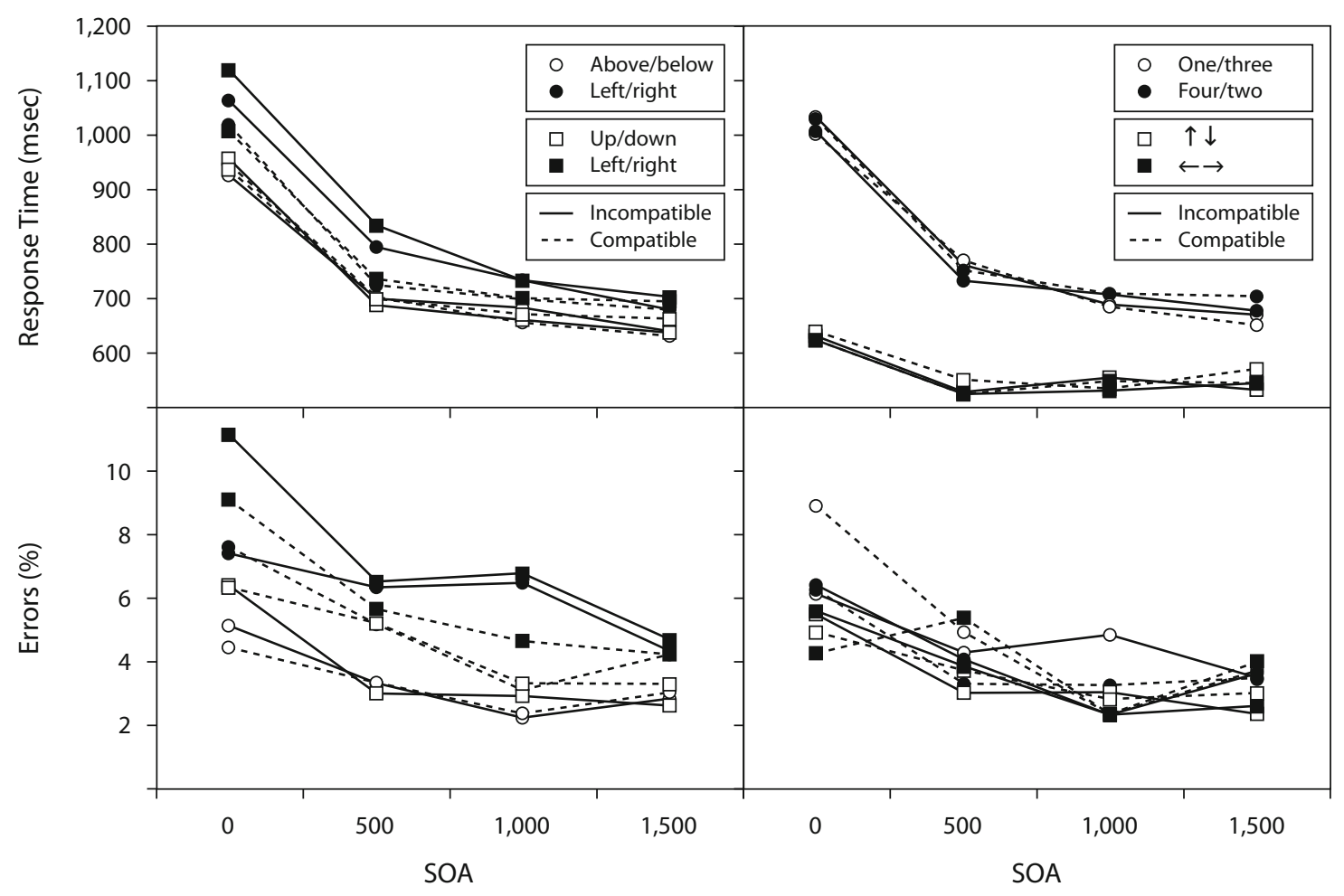

Figure 5. Mean correct response times (top panels) and error rates in percentages (bottom panels) depicted as function of cued location, opposite compatibility, and stimulus onset asynchrony (SOA) in Experiment 2. The "above/below/left/ right" and "up/down/left/right" cue conditions are depicted on the left side of the figure, and the "one/three/four/two" and " $\uparrow / \downarrow / \leftarrow / \rightarrow$ " cue conditions are depicted on the right side of the figure. 
This analysis revealed significant main effects of opposite compatibility $\left[F(1,70)=28.31, p<.001, \eta_{\mathrm{p}}^{2}=.29\right]$ and $\mathrm{SOA}\left[F(3,210)=474.34, p<.0001, \eta_{\mathrm{p}}^{2}=.87\right]$. In addition, this analysis revealed a significant opposite compatibility $\times$ SOA interaction $[F(3,210)=7.78, p<.001$, $\left.\eta_{\mathrm{p}}^{2}=.10\right]$. As in Experiment 1, this interaction indicated that the magnitude of the opposite compatibility effect decreased as SOA increased (subsequent analyses revealed that the opposite compatibility effect was significant in the 0-, 500-, and 1,000-msec SOA conditions; all $p$ s $<$ $.05)$. However, none of the interactions involving experiment approached significance (all $p \mathrm{~s}>.25$ ).

The next analysis compared the opposite compatibility effect across the "up/down" and "above/below" conditions in Experiment 2. This analysis revealed that the present paradigm does not appear to be sensitive to differences in the complexity of the spatial relations or direction specifications that are expressed by "up/down" and "above/ below." This conclusion was supported by a nonsignificant main effect of opposite compatibility $[F(1,34)<1]$ and a nonsignificant opposite compatibility $\times$ cue type interaction $[F(1,34)=1]$. In addition, it is important to note that, overall, RTs were very similar across the "up/down" $(M=738 \mathrm{msec})$ and "above/below" $(M=735 \mathrm{msec})$ conditions, and the main effect of cue type did not approach significance $[F(1,34)<1]$.

The last analysis conducted on mean correct RT evaluated the opposite compatibility effect in each of the number word and arrow conditions. This analysis revealed that the opposite compatibility effect did not attain significance in any of the four cue type $\times$ cued location conditions (all $p \mathrm{~s}>.05$ ). In addition, it should be noted that the nonsignificant effect of opposite compatibility observed in the number word condition provides evidence against an alternative, scanning account of the effect observed in the spatial word condition. Had the significant effect of opposite compatibility observed in the spatial word condition been due to left-right scanning associated with linguistic processing, a similar effect should have been observed in response to "four/two" cues in the number word condition.

Furthermore, it should also be noted that the nonsignificant effect of opposite compatibility observed in the number word condition also provides evidence against an alternative, left-right confusion account of the effect observed in the spatial word condition. Had the significant effect of opposite compatibility observed in the spatial word condition been due to left-right confusion, a similar effect should have been observed in response to "four/two" cues in the number word condition, because the spatial meaning of those cues was defined in terms of left and right.

An identical four-way, mixed ANOVA was also performed on error rates (in percentages). The pattern of error rates generally mirrored the pattern of mean correct RTs. The statistical analysis revealed significant main effects of cued location $\left[F(1,68)=15.29, p<.001, \eta_{\mathrm{p}}^{2}=.18\right]$ and $\mathrm{SOA}\left[F(3,204)=33.47, p<.001, \eta_{\mathrm{p}}^{2}=.33\right]$. In addition, the statistical analysis revealed a significant cue type $X$ cued location interaction $\left[F(3,68)=8.12, p<.001, \eta_{\mathrm{p}}^{2}=\right.$
.26], indicating a significant effect of cued location in the two spatial word conditions, but not in the other two cue conditions. Thus, the present pattern of findings does not appear to reflect a speed-accuracy trade-off.

\section{GENERAL DISCUSSION}

Humans routinely use a variety of directional symbols to control the spatial distribution of attention. In so doing, spatial information may be communicated from one individual to another across opposing frames of reference, which in turn can lead to inconsistent mappings between symbols and directions (or locations). Some symbols, such as "left/right," can refer to the opposite direction when these symbols are encountered from opposing perspectives, whereas other symbols, such as " $\leftarrow / \rightarrow$," typically refer only to the cued direction. Such observations led to the hypothesis that, over time, "left/right" cues may be experienced as providing less valid spatial information across contexts than do, for example, " $\leftarrow / \rightarrow$ " cues, which in turn might influence the manner in which attention is distributed in space (Johnson \& Yantis, 1995). Consistent with this differential validity hypothesis, the present study has provided important evidence that not all directional symbols can focus attention equally well across the visual field.

More specifically, the findings obtained in the visual selection task (when ambiguous target displays were shown) suggested that the identity of the opposite circle had a greater influence on performance when "left/right" cues were used to specify cued location along the horizontal axis than when "above/below" or "up/down" cues were used to specify cued location along the vertical axis. In addition, the identity of the opposite circle had relatively little influence on performance when " $\leftarrow / \rightarrow$ " and "four/ two" cues were used to specify cued location along the horizontal axis. These findings are important because they demonstrate that weaker selection is tied to the learned validity of the directional symbols and is not intrinsic to the horizontal axis or to the selective mechanisms that are directed along it. Likewise, the findings obtained in the probe detection task (when unambiguous target displays were shown) corroborated the findings obtained in the visual selection task and suggested that selective attention was also less focused when "left/right" cues were used to specify the cued location along the horizontal axis than when "above/below" cues were used to specify the cued location along the vertical axis.

Altogether, the present findings reveal an important semantic-based constraint on the focus of selective attention that has a number of important implications for theories of symbolic control. First, there has been considerable interest in understanding the cognitive and neurobiological mechanisms underlying the symbolic control of attention (see, e.g., Corbetta, Kincade, Ollinger, \& Shulman, 2000; Dosher \& Lu, 2000; Gibson \& Kingstone, 2006; Goldberg, Maurer, \& Lewis, 2001; Hommel et al., 2001; Hopfinger, Buonocore, \& Mangun, 2000; Johnson, McGrath, \& McNeil, 2002; Jonides, 1981; Nobre, 
Sebestyen, \& Miniussi, 1995; Posner et al., 1980; Ristic \& Kingstone, 2006). However, the vast majority of these previous studies have used only a single type of directional symbol - the arrow - to study the symbolic control of attention. As a result, very little is known about how variation in symbolic processing may interact with attentional processing. The present findings are important because they suggest that different types of directional symbols convey spatial information in different ways and that such semantic variation can influence the spatial distribution of selective attention. Thus, theories of symbolic control can no longer ignore the diversity of processing that underlies the semantics of spatial symbols. Furthermore, by showing differences in the focus of selection, the present findings also have practical implications for understanding how to design potentially safer navigational systems (see, e.g., Ho \& Spence, 2006).

Second, the present findings are also relevant to recent studies of symbolic control that have investigated whether directional symbols can elicit involuntary shifts of attention (Friesen, Ristic, \& Kingstone, 2004; Gibson \& Bryant, 2005; Ho \& Spence, 2006; Hommel et al., 2001; Ristic, Friesen, \& Kingstone, 2002; Tipples, 2002, 2008). These findings reveal that observers often have difficulty ignoring the spatial information that is conveyed by directional symbols, even when that information is no longer valid within the confines of the laboratory. However, these previous studies have focused exclusively on the question of whether previously learned directional symbols can elicit involuntary shifts of attention to cued locations. In contrast, the findings obtained in the present study suggest that certain previously learned spatial terms, such as "left" and "right," may also elicit involuntary shifts of attention to uncued (opposite) locations as well.

More specifically, the observers never had any incentive to attend to the opposite location in the present study. Nevertheless, the present findings revealed that the observers could not ignore the opposite location when "left/ right" cues were shown, suggesting that such counterorienting may have been involuntary. In considering the nature of the present findings, it is important to point out that these spatial effects did not arise simply because the observers were unsure of where the left and right cued locations were in the present experiments. Such uncertainty was diminished in the present study because the specification of all four cued locations remained stable across the duration of the experiments and all were consistently defined relative to the observer's canonical frame of reference. Indeed, findings obtained when " $\leftarrow / \rightarrow$ " and "four/ two" cues were shown indicated that the observers could ignore the horizontally specified opposite locations under some conditions.

In order to further characterize the nature of these effects, we conducted two subsequent analyses including the 72 observers from Experiments 1 and 2 that served in the "left/right" condition. In the first analysis, we divided the experimental trials into three equal blocks and investigated whether the magnitude of the opposite compatibility effect decreased over the course of the experiment. If cue processing involved automatic activation of both the cued and opposite locations in the "left/right" cue condition, the effects of this activation should remain relatively stable throughout the duration of the experiment. The results were consistent with this expectation: None of the interactions involving blocks approached significance (all $p \mathrm{~s}>.15$ ). For instance, the magnitude of the opposite compatibility effect was found to be $83 \mathrm{msec}$ in Block 1 , $72 \mathrm{msec}$ in Block 2, and $89 \mathrm{msec}$ in Block 3 when the SOA was $0 \mathrm{msec}$ (the SOA at which the opposite compatibility effect was observed to be largest).

In the second analysis, we created cumulative RT distributions for each of the 72 observers, using the Vincentizing procedure (Ratcliff, 1979). In addition, we focused this analysis on the 0 -msec SOA condition, because the magnitude of the opposite compatibility effect tended to decrease as a function of SOA. Of particular interest was whether the opposite compatibility effect would be observed across the whole RT distribution or whether this effect would be localized to only one part of the RT distribution or another. If cue processing involved fast and automatic activation of both the cued and opposite locations in the "left/right" cue condition, the effects of this activation should be observable throughout the whole RT distribution.

Figure 6 shows the magnitude of the opposite compatibility effect plotted as a function of cumulative RT percentiles. As can be seen, the magnitude of the opposite compatibility effect increased gradually as cumulative RT percentages increased up to the 90th percentile and then increased abruptly in the longest RT bin. Because exposure to the target display increased as a function of RT bin, the increase in the magnitude of the opposite compatibility effect as a function of RT bin is not surprising and likely reflects increased exposure to the distractor (Lavie $\&$ de Fockert, 2003). Most important, the opposite compatibility effect was found to be significant across all 10 RT conditions (all $p \mathrm{~s}<.05$ ), indicating that the color of the opposite circle had a significant effect on performance across the whole distribution of RTs.

Such findings are consistent with the notion that spatial cues such as "left" do, indeed, automatically activate both

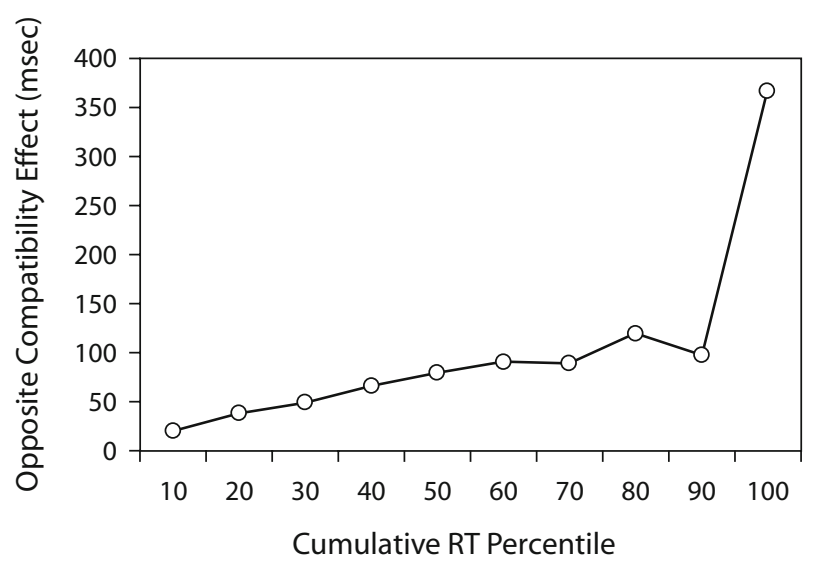

Figure 6. Mean opposite compatibility effect (incompatible response time [RT] - compatible RT) averaged across the "left/ right" cue conditions in Experiments 1 and 2, depicted as a function of cumulative RT percentile. 
the left and the right cued locations (to varying degrees). This conclusion was further clarified by the cue validity effects observed in the probe detection task of Experiment 1 . The significant cue validity effects observed in the "left/right" cue condition suggested that the cued location was activated more than any of the uncued locations that could be specified along the horizontal axis. In addition, the similar results observed in both the invalid-opposite and invalid-fixation conditions suggested that attention may have been divided along this axis, rather than shifted in a discrete fashion to just the opposite and cued locations. Hence, the present findings extend previous findings suggesting that "left/right" cues can elicit involuntary shifts of attention to the cued location (Ho \& Spence, 2006; Hommel et al., 2001) by suggesting that they can elicit a weaker involuntary distribution of attention along the uncued portions of the horizontal axis as well.

Third, the present findings are also relevant to theories of symbolic control that have attempted to explain the sole occurrence of the cued location effect in the word cue condition in terms of the complexity of spatial relations expressed, together with the notion that the vertical axis can be accessed more efficiently than the horizontal axis (Gibson \& Kingstone, 2006; Logan, 1995). Although the differential validity hypothesis can coexist with the spatial relations hypothesis, it is important to note that the spatial relations hypothesis cannot explain the differential effect of opposite compatibility or cue validity that was observed in the present study. Moreover, comparison of the "up/down" and "above/below" cue conditions in Experiment 2 failed to support a role for relational complexity, even though "up/down" cues were hypothesized to express a more basic spatial relation than did "above/ below" cues. Thus, it is possible that the RT difference observed in previous studies (Gibson \& Kingstone, 2006; Logan, 1995) between the left/right and above/below cued location conditions might be due solely to the differential validity of the cues.

Note also that the present findings are consistent with an experiment conducted by Logan (1995; see Experiment 10) in which "left" and "right" spatial word cues were defined relative to an image of a human head that was sometimes oriented in the same direction as the observer (the $0^{\circ}$ top view condition) and sometimes oriented in the opposite direction as the observer (in the $0^{\circ}$ front view condition). As in the present experiment, Logan's (1995) results could be examined with respect to the compatibility of the items appearing in the cued and opposite locations (see Table 3 in Logan, 1995). Examination of his results suggested that the magnitude of the compatibility effect was approximately twice as large when direction was defined so as to be opposite to the observer's own frame of reference than when direction was defined so as to be consistent with the observer's own frame of reference; however, a specific statistical test of this interaction was not conducted. These findings generally corroborate the present findings, using a more complex configuration, and suggest that the color of the opposite circle has a stronger effect when the word cue is defined so as to be consistent with the observer's own frame of reference.
However, Logan (1995) interpreted his opposite compatibility findings as supporting an axis-based account. In particular, Logan (1995) argued that shorter RTs in the compatible condition, relative to the incompatible condition, suggest that the orientation of the reference frame can be computed separately from the direction. However, according to Logan's (1995) account, a similar compatibility effect should also be observed in response to "above" and "below" cues, but no effect of compatibility is apparent from Logan's (1995) findings when these cued locations are considered, although the significance of this effect was not directly tested in Logan's (1995) study. Thus, altogether, the findings appear to be more consistent with the differential validity account than with the axis-based account.

One limitation of existing studies of symbolic control is that they have typically used well-learned directional symbols, such as words and arrows, to cue attention (Corbetta et al., 2000; Dosher \& Lu, 2000; Gibson \& Kingstone, 2006; Goldberg et al., 2001; Hommel et al., 2001; Hopfinger et al., 2000; Johnson et al., 2002; Jonides, 1981; Nobre et al., 1995; Posner et al., 1980; Ristic \& Kingstone, 2006). As a result, the observed cuing effects reflect semantic information that is, by definition, established outside the context of the experiment. Likewise, in the present study, prior experience with words and arrows played a critical role in understanding the functional differences that were observed between the "left/right" and the " $\leftarrow / \rightarrow$ " cues. However, because differential validity was not explicitly manipulated within the context of the experiment, there may be lingering doubt that some other, uncontrolled difference between words and arrows caused the observed functional differences. The finding that novel "four/two" cues could reproduce the cuing effects observed with " $\leftarrow l \rightarrow$ " cues while prior experience is controlled provides some reassurance that efficient selection is not a simple artifact. Future studies can provide similar reassurance that the inefficient selection observed in response to "left/ right" cues is not a simple artifact by training inconsistent mappings between number word cues and cued directions. Of particular interest will be whether such ambiguity can be induced simply by stipulating different mappings (e.g., "four" means left for the first 100 trials but it means right for the next 100 trials), or whether such ambiguity depends on the presence of opposing frames of reference.

\section{AUTHOR NOTE}

The authors thank Adam Biggs, Bradley A. Dobrzenski, and Ann C. Flies for their help collecting the data for the present experiments. Correspondence concerning this article should be addressed to B. S. Gibson, Department of Psychology, University of Notre Dame, 118 Haggar Hall, Notre Dame, IN 46556 (e-mail: bgibson@nd.edu).

\section{REFERENCES}

Bryant, D. J., TVERSky, B., \& Franklin, N. (1992). Internal and external spatial frameworks for representing described scenes. Journal of Memory \& Language, 31, 74-98.

CARLson, L. A. (2003). Using spatial language. In B. H. Ross (Ed.), The psychology of learning and motivation (Vol. 43, pp. 127-161). San Diego: Academic Press.

Clark, H. H. (1973). Space, time, semantics, and the child. In T. E. 
Moore (Ed.), Cognitive development and the acquisition of language (pp. 27-63). New York: Academic Press.

Corballis, M. C. (1988). Recognition of disoriented shapes. Psychological Review, 95, 115-123.

Corbetta, M., Kincade, M., Ollinger, J. M., McAvoy, M. P., \& Shulman, G. L. (2000). Voluntary orienting is dissociated from target detection in human posterior parietal cortex. Nature Neuroscience, 3, 292-297.

Coventry, K. R., \& Garrod, S. C. (2004). Saying, seeing, and acting: The psychological semantics of spatial prepositions. Hove, U.K.: Psychology Press.

Dosher, B. A., \& LU, Z. L. (2000). Noise exclusion in spatial attention. Psychological Science, 11, 139-146.

Franklin, N., \& TVERSKy, B. (1990). Searching imagined environments. Journal of Experimental Psychology: General, 119, 63-76.

Friesen, C. K., Ristic, J., \& Kingstone, A. (2004). Attention effects on counterpredictive gaze and arrow cues. Journal of Experimental Psychology: Human Perception \& Performance, 30, 319-329.

Gibson, B. S., \& BRyANT, T. A. (2005). Variation in cue duration reveals top-down modulation of involuntary orienting to uninformative symbolic cues. Perception \& Psychophysics, 67, 749-758.

Gibson, B. S., \& Kingstone, A. (2006). Visual attention and the semantics of space: Beyond central and peripheral cues. Psychological Science, 17, 622-627.

Goldberg, M., Maurer, D., \& Lewis, T. (2001). Developmental changes in attention: The effects of endogenous cuing and of distractors. Developmental Science, 4, 209-219.

Ho, C., \& Spence, C. (2006). Verbal interface design: Do verbal directional cues automatically orient visual spatial attention? Computers in Human Behavior, 22, 733-748.

Hommel, B., Pratt, J., Colzato, L., \& Godijn, R. (2001). Symbolic control of visual attention. Psychological Science, 12, 360-365.

Hopfinger, J., Buonocore, M., \& MAngun, G. (2000). The neural mechanisms of top-down attentional control. Nature Neuroscience, 3, 284-291.

Johnson, D. N., McGrath, A., \& McNeil, C. (2002). Cuing interacts with perceptual load in visual search. Psychological Science, 13, 284-287.

Johnson, D. N., \& Yantis, S. (1995). Allocating visual attention: Tests of a two-process model. Journal of Experimental Psychology: Human Perception \& Performance, 21, 1376-1390.

JonIDES, J. (1981). Voluntary versus automatic control over the mind's eye movement. In J. [B.] Long \& A. [D.] Baddeley (Eds.), Attention and performance IX (pp. 187-203). Hillsdale, NJ: Erlbaum.

JONIDES, J., \& MACK, R. (1984). On the cost and benefit of cost and benefit. Psychological Bulletin, 96, 29-44.

Kemmerer, D. (2006). The semantics of space: Integrating linguistic typology and cognitive neuroscience. Neuropsychologia, 44, 1607-1621.

KuČERA, H., \& Francis, W. N. (1967). Computational analysis of present-day American English. Providence, RI: Brown University Press.

LAVIE, N., \& DE FockerT, J. W. (2003). Contrasting effects of sensory limits and capacity limits in visual selective attention. Perception \& Psychophysics, 65, 202-212.

Lavie, N., Hirst, A., DE Fockert, J. W., \& Viding, E. (2004). Load theory of selective attention and cognitive control. Journal of Experimental Psychology: General, 133, 339-354.
Levinson, S. C. (2003). Space in language and cognition. Cambridge: Cambridge University Press.

LoGAN, G. D. (1994). Spatial attention and the apprehension of spatial relations. Journal of Experimental Psychology: Human Perception \& Performance, 20, 1015-1036.

LoGAN, G. D. (1995). Linguistic and conceptual control of visual spatial attention. Cognitive Psychology, 28, 103-174.

LoGAN, G. D. (1996). Top-down control of reference frame alignment in directing attention from cue to target. In A. F. Kramer, M. G. H. Coles, \& G. D. Logan (Eds.), Converging operations in the study of visual selective attention (pp. 415-438). Washington, DC: American Psychological Association.

Logan, G. D., \& SAdLER, D. D. (1996). A computational analysis of the apprehension of spatial relations. In P. Bloom, M. A. Peterson, L. Nadel, \& M. Garrett (Eds.), Language and space (pp. 493-529). Cambridge, MA: MIT Press.

Mayer, A. R., \& Kosson, D. S. (2004). The effects of auditory and visual linguistic distractors on target localization. Neuropsychology, 18, 248-257.

Nobre, A., Sebestyen, G., \& Miniussi, C. (1995). The dynamics of shifting visuospatial attention revealed by event-related potentials. Neuropsychologia, 38, 964-974.

PASHLER, H. (1998). The psychology of attention. Cambridge, MA: MIT Press.

Posner, M. I. (1980). Orienting of attention. Quarterly Journal of Experimental Psychology, 32, 3-25.

Posner, M. I., SNyder, C. R., \& Davidson, B. J. (1980). Attention and the detection of signals. Journal of Experimental Psychology: General, 109, 160-174.

Prinzmetal, W., McCool, C. K., \& Park, S. (2005). Attention: Reaction time and accuracy reveal different mechanisms. Journal of Experimental Psychology: General, 134, 73-92.

RATCLIFF, R. (1979). Group reaction time distributions and an analysis of distribution statistics. Psychological Bulletin, 86, 446-461.

Ristic, J., Friesen, C., \& Kingstone, A. (2002). Are the eyes special? It depends on how you look at it. Psychonomic Bulletin \& Review, 9 , 507-513.

Ristic, J., \& Kingstone, A. (2006). Attention to arrows: Pointing to a new direction. Quarterly Journal of Experimental Psychology, 59, 1921-1930.

SCHOBER, M. F. (1993). Spatial perspective-taking in conversation. Cognition, 47, 1-24.

Schober, M. F. (1995). Speakers, addressees, and frames of reference: Whose effort is minimized in conversations about locations? Discourse Processes, 20, 219-247.

TipPLES, J. (2002). Eye gaze is not unique: Automatic orienting in response to uninformative arrows. Psychonomic Bulletin \& Review, 9, 314-318.

TIPPLES, J. (2008). Orienting to counterpredictive gaze and arrow cues. Perception \& Psychophysics, 70, 77-87.

van der HeIJden, A. H. C. (1992). Selective attention in vision. New York: Routledge.

Vecera, S. P., \& Rizzo, M. (2004). What are you looking at? Impaired "social attention" following frontal-lobe damage. Neuropsychologia, 42, 1657-1665.

(Manuscript received May 13, 2008 revision accepted for publication August 24, 2008.) 